\title{
Parallel processing of part-whole information in visual search tasks
}

\author{
JEREMY M. WOLFE \\ Brigham and Women's Hospital, Boston, Massachusetts \\ and Harvard Medical School, Boston, Massachusetts \\ STACIA R. FRIEDMAN-HILL \\ University of California, Davis, California \\ and \\ ALEXANDER B. BILSKY \\ Brigham and Women's Hospital, Boston, Massachusetts
}

\begin{abstract}
Combination of information from the parallel processing of different basic features (color, size, etc.) can be used to guide attention to targets defined by conjunctions of those features. Wolfe et al. (1990) argued that, although it was possible to guide attention to the conjunction of, for instance, color and orientation, it was not possible to guide attention to targets defined by conjunctions of two colors or two orientations. The present experiments demonstrate an exception to this rule. Although it is true that attention cannot be guided to a target that has red and green parts, attention can be guided to an item that can be described as a whole red target with a green part. In Experiments 1 and 2, we illustrate this point. In Experiments 3 and 4, we rule out some simple size-based accounts of this finding. In Experiments 5 and 6, we begin to explore the nature of these first steps toward dividing the visual scene into parts and wholes.
\end{abstract}

In visual search experiments, subjects search for a target item among distractor items. In one standard paradigm, used here, the target is presented among a variable number of distractor items, reaction time (RT) is measured, and the slope of RT $\times$ set size functions is computed. Search is guided by the output of a preattentive stage of visual processing that can be described as a set of feature modules. Each of these modules can process information about a single basic feature (color, size, orientation, etc.) in parallel across large portions of the visual field (see Lennie, Trevarthen, VanEssen, \& Wassle, 1990; Treisman, 1986b, for reviews). The early stages of preattentive processing are spatiotopic, registering the presence of redness or of verticality at some location in space. However, it seems clear that visual search is not a search for some collection of features but a search for an item or object with those features (Kahneman \& Treisman, 1984; Tipper, Brehaut, \& Driver, 1990; Tipper, Driver, \& Weaver, 1991; Treisman, 1986a, 1988). In this paper, we examine how basic featural information is attached to the objects or items.

\footnotetext{
We thank Patricia O'Neill, Nancy Kanwisher, and an anonymous reviewer for comments on drafts of this paper. This research is supported by NIH-NEI Grant ROI-EY05087 and AFOSR Grant F49620. S.R.F.-H. is affiliated with the Center for Neuroscience at UCD; A.B.B. is with the Center for Ophthalmic Research at Brigham and Women's Hospital. Correspondence concerning this article should be addressed to J. M. Wolfe, Center for Ophthalmic Research, Brigham and Women's Hospital, 221 Longwood Ave., Boston, MA 02115.
}

The specific experiments reported here deal with the color of items. Visual search for an item of one color among distractors of another color is very efficient as long as there is a sufficient difference between the colors (Nagy $\&$ Sanchez, 1990). The word efficient, as it is used here, means that the slopes of RT $\times$ set size functions are near zero. Search remains efficient even when the distractor set contains items of more than one color (Duncan, 1989), again with the constraint that the colors be properly placed in color space (D'Zmura, 1991). Reasonably efficient visual search is possible for conjunctions of color with other basic features (e.g., color $\times$ orientation-search for a red vertical item among red horizontal and green vertical distractors). Such search tasks produce average slopes of 6-12 msec/item on target-present trials (Wolfe, Cave, \& Franzel, 1989). However, subjects cannot search efficiently for items defined by the conjunction of two colors (e.g., find the item that is red and green among distractors that are either red and blue or blue and green). A variety of these color $\times$ color conjunctions produced average target trial slopes in the range of 20-60 msec/item and blank trial slopes of about twice those for the target trials, consistent with a slow, serial self-terminating search (Wolfe et al., 1990).

The efficiency of search tasks can be understood as a function of the ability of the output of parallel feature processes to guide attention toward the target (Cave \& Wolfe, 1990; Egeth, Virzi, \& Garbart, 1984; Wolfe et al., 1989; Zohary \& Hochstein, 1989). In the simplest case, distractors are homogeneous and the target is uniquely de- 
fined by the basic feature. In this case, a large attentionattracting signal is always generated at the location of the target and attention is guided to the target before it can be deployed elsewhere. Reaction times are independent of the number of distractors. In cases in which the differences between target and distractors are not so large or when distractors are inhomogeneous, the useful signal generated by the target may be smaller than the noisy signals generated by some of the distractors (Chun \& Wolfe, 1994; Pavel, Econopouly, \& Landy, 1992). If attention is deployed from item to item in the decreasing order of the strength of these signals, the target will not be the first item to attract attention and RTs will increase. The larger the set size, the larger the number of high-activation distractors and the greater the increase in RT. In the case in which the parallel processes generate no guiding signals, search proceeds randomly in a serial, self-terminating fashion.

Information from two or more parallel processes can be combined in the guidance of attention. This allows for the efficient search for targets defined by conjunctions of basic features. Thus, in a search for a red vertical item among red horizontal and green vertical items, a parallel color processor can add signal strength to all red items while a parallel orientation processor adds signal strength to all vertical items. Even in the presence of internal noise, the sum of these signals will tend to guide attention toward the red vertical items. According to this guided search model (Wolfe, 1992, 1994), this combination of information yields the near "parallel" searches for conjunctions recently reported (Dehaene, 1989; Egeth et al., 1984; McLeod, Driver, \& Crisp, 1988; Nakayama \& Silverman, 1986; Quinlan \& Humphreys, 1987; Treisman \& Sato, 1990; Wolfe et al., 1989; Wolfe, Yee, \& Friedman-Hill, 1992).

The color $x$ color tasks described above are a class of conjunction search tasks in which no guidance appears to have been possible. In previous work, not only color $x$ color but orientation $x$ orientation and size $\times$ size conjunctions produced very inefficient searches, yielding steeper RT $\times$ set size functions than most standard "serial" searches. Wolfe et al. (1990) concluded that guidance was not possible for within-feature conjunctions because it was not possible for one feature module to handle two requests separately at the same time. Apparently, the color module could not add the red and the green "signals" to find a red and green item in the same way that it could add red and vertical signals to find red-vertical items.

In this paper, we report on an exception to the rule that guidance is not possible for within-feature conjunctions. Specifically, attention can be guided to a two-colored item when the relationship between the colored regions is hierarchical. That is, although it is true that attention cannot be guided to the item that is red and green, attention can be guided to the red item with the green on it. Apparently, for purposes of the preattentive guidance of visual search, an item cannot be red and green, but a red item can have green on it. If search can be guided on the basis of the hierarchical relationship of patches of color, it follows that the figural basis of that hierarchical relationship must be extracted in parallel, prior to the serial deployment of attention. We will refer to that hierarchical relationship between colored patches as the relationship between parts and wholes. Subjects found the part-whole distinction to be a natural way to describe the stimuli (e.g., in Experiment 1-" "Find the red house with the yellow windows"). We use these terms in a very restricted manner, to refer to a hierarchical relationship between features in an item in a visual search task. More colloquial use of a term like whole undoubtedly implies perceptual processing that we would not wish to attribute to the preattentive stage. We will return to the issue of the actual preattentive representation in the discussion section. ${ }^{1}$

If these part-whole terms are taken in our restricted sense, Experiments 1 and 2 demonstrate that conjunction search for a whole item of one color with a part of another color is more efficient than conjunction search for an item with parts of two colors. Experiments 3 and 4 rule out some simple size-based accounts of this finding. Experiment 5 shows that a region is more likely to be treated as a "part" if it is surrounded by another region. Finally, Experiment 6 shows that the definitions of "parts" and "wholes" can be task specific. A "whole" in one task can be a "part" in another.

\section{EXPERIMENT 1 \\ Conjunctions of the Color of a Whole With the Color of a Part}

As noted above, Wolfe et al. (1990) found visual search for conjunctions of two colors to be very inefficient. Indeed, a characteristic of within-feature searches of this sort is that they are even more inefficient (i.e., they have steeper RT $\times$ set size slopes) than other well-established "serial"' searches (e.g., to search for a T among Ls, with variable orientation of the Ts and Ls; see Kwak, Dagenbach, \& Egeth, 1991). In all of the color $\times$ color search experiments in Wolfe et al. (1990), subjects searched for two-colored items in which the two colors had no hierarchical relationship to one another. In Experiment 1, we show that search is much easier when the stimuli can be characterized in hierarchical terms as whole items of one color with parts of another color.

\section{Method}

Stimuli. Examples of the stimuli are shown in Figure 1. In all conditions, the target was red and yellow. Half of the distractors were red and blue, and the other half, blue and yellow. In the partpart version, these stimuli were "two-family" houses with one half painted one color, and the other half, the other color. In the part-whole version, the stimuli were "single-family" houses painted one color with windows of another color. In the feature search control condition, the stimuli were two-family houses and the target was the color yellow, displayed only in the small windows. At the $62-\mathrm{cm}$ viewing distance, the houses subtended $2.3^{\circ} \times 1.4^{\circ}$. The windows subtended $0.3^{\circ} \times 0.5^{\circ}$. For Experiments 1 and 2, CIE coordinates and luminance values for stimuli were as follows: red, 


\section{EXPERIMENT ONE: STIMULI}

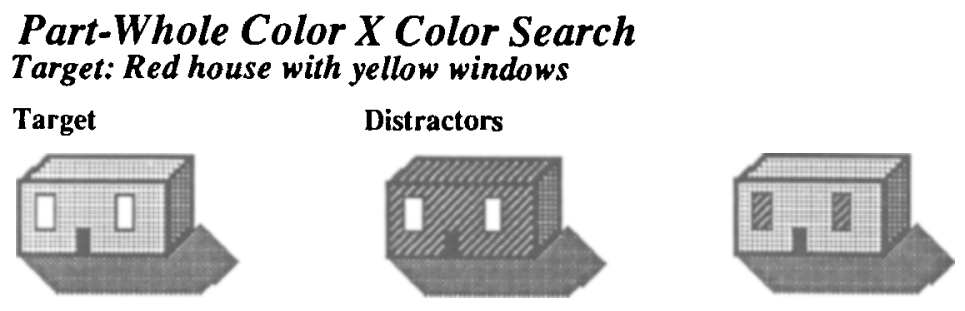

\section{Part-Part Color X Color Search Target: Red \& yellow house}
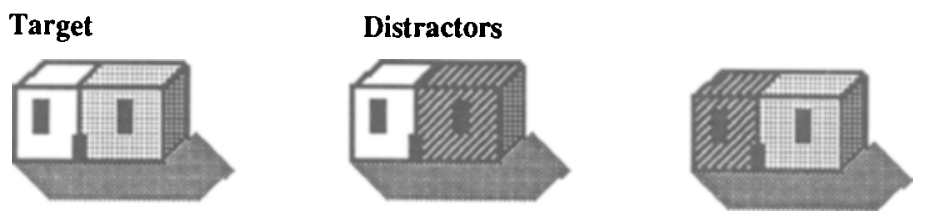

\section{Color Feature Search Target: Yellow windows}
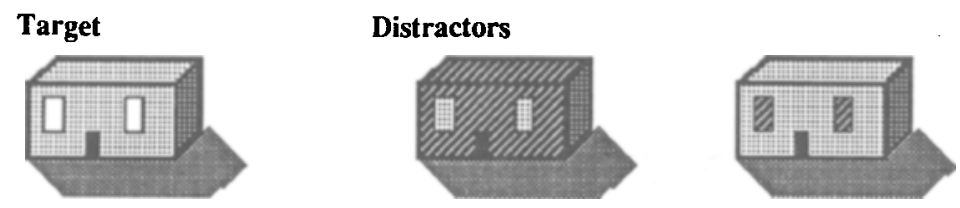

Figure 1. Stimuli for Experiment 1.

$x=.532, y=.333$, lum $=9.6 \mathrm{~cd} / \mathrm{m}^{2} ;$ yellow, $x=.423, y=$ .485 , lum $=33.5 \mathrm{~cd} / \mathrm{m}^{2}$; green, $x=.278, y=.560$, lum $=$ $19.9 \mathrm{~cd} / \mathrm{m}^{2}$; blue, $x=.192, y=.223$, lum $=23.0 \mathrm{~cd} / \mathrm{m}^{2}$. Black and white renditions of the stimuli are found in Figure 1. Relevant information is described below.

Part-whole color $\times$ color. Target: red house with yellow windows. Distractors: red houses with blue windows, and blue houses with yellow windows.

Part-part color $\times$ color. Target: red and yellow two-family house. Distractors: red and blue two-family houses, and blue and yellow two-family houses. Red was always on the left, yellow on the right. Blue was to the right of red in one distractor and to the left of yellow in the other.

Color feature search. Target: red house with yellow windows. Distractors: red houses with blue windows, and blue houses with red windows. Subjects searched for yellow windows.

The stimuli were presented on a Macintosh II computer using VSearch software (Enns, Ochs, \& Rensink, 1990). Set sizes of 4, 10 , and 16 items were used. These items were presented in random locations in a jiggled $5 \times 4$ array of possible locations. The stimulus array subtended an area $13.6^{\circ} \times 13.6^{\circ}$ at the $62-\mathrm{cm}$ viewing distance. Background luminance was $24.6 \mathrm{~cd} / \mathrm{m}^{2}$. White regions of the figures were $39.1 \mathrm{~cd} / \mathrm{m}^{2}$. The luminance of the black regions was not measurable. The stimuli were presented for a maximum of $3,000 \mathrm{msec}$. The subjects responded with a keypress to indicate target presence or absence. Response accuracies and RTs were recorded. Accuracy feedback was given after each trial. Each condition was run in a separate block. The subjects were tested for 30 practice and 300 experimental trials in each condition. The order of conditions was pseudorandom across subjects. Set size and target presence were random across trials within a condition.

Subjects. Ten volunteer subjects, 18 to 35 years of age, were tested. All had acuity of at least 20/25 and normal color vision (Dvorine plates). All gave informed consent and were paid for their participation.

\section{Results}

Average RT $\times$ set size functions are shown in Figure 2 (top panel). A scatterplot of target-present versus targetabsent slopes is given in Figure 2 (bottom panel). A classic serial search would produce target trial slopes of 20$30 \mathrm{msec} /$ item and blank trial slopes of $40-60 \mathrm{msec} / \mathrm{item}$. It is obvious that the part-whole color $\times$ color conjunction searches are far easier than the part-part versions of the same task and are shallower than classic serial search slopes. Average part-whole slopes are 13.1 and $23.7 \mathrm{msec} /$ item for target and blank trials, respectively. This is significantly shallower than the 38.9 and $67.4 \mathrm{msec} / \mathrm{item}$ average slopes in the part-part condition $[t(9)>8.4, p<$ .0001 , for each comparison]. The scatterplot data for the part-whole version (open circles) suggests that 3 subjects executed something resembling a serial, self-terminating search, with the other 7 showing much more efficient search. The color feature search yielded average slopes of 1.9 and $0.3 \mathrm{msec} /$ item. 


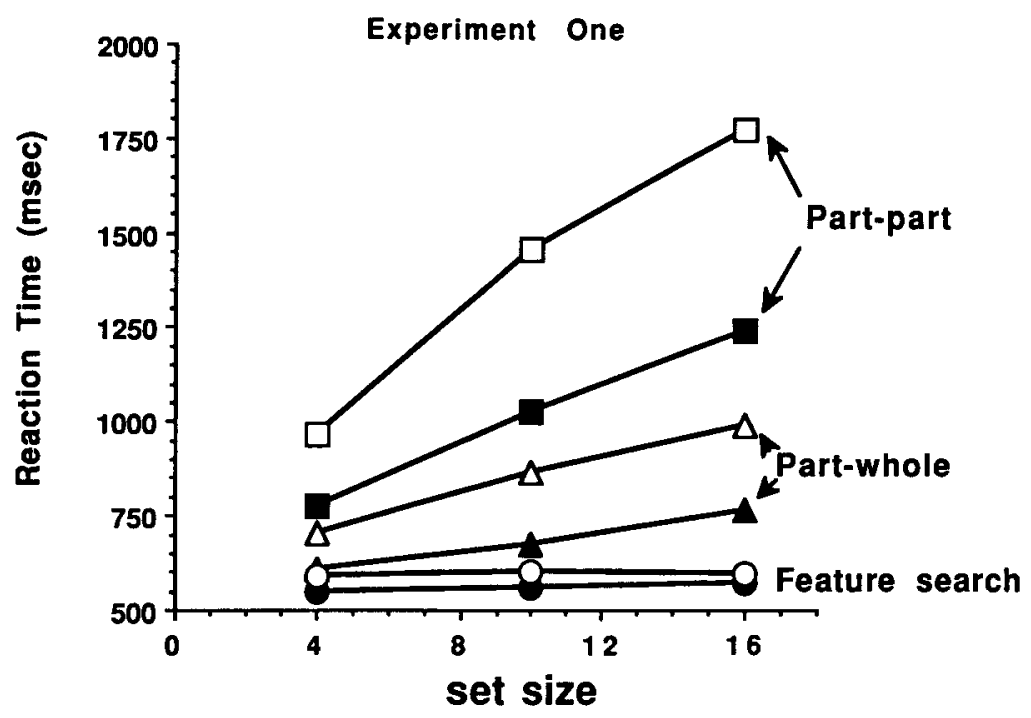

Subject Slopes For Experiment One

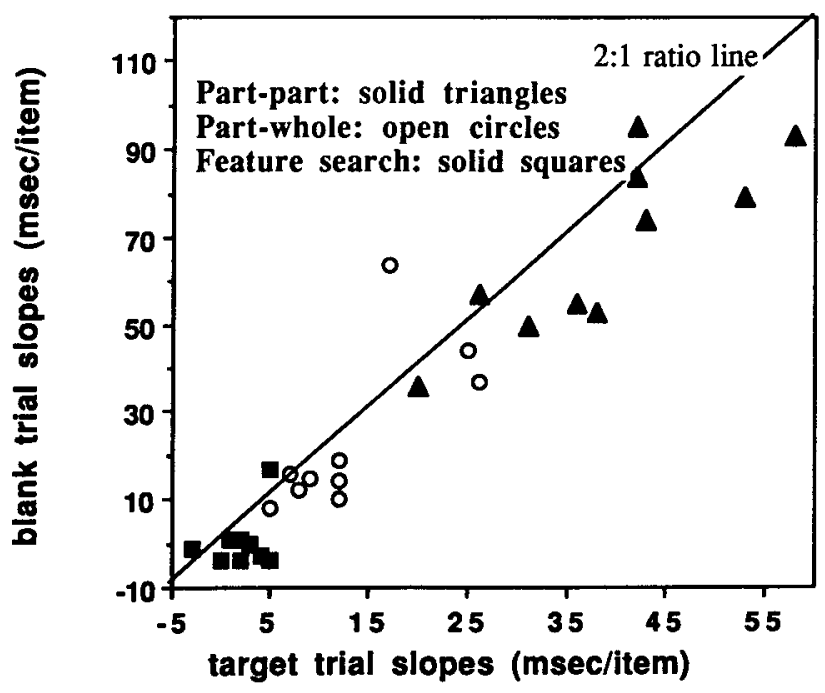

Figure 2. Results for Experiment 1, showing that the part-whole condition produces much more efficient search than does the part-part condition. Part-part slopes are 38.9 msec/item for the target-present trials and $67.4 \mathrm{msec} /$ item for the target-absent trials. This can be compared to 13.1 and 23.7 , respectively, for the part-whole case and 1.9 and 0.3 for the feature case. Lower panel shows a scatterplot of individual slopes indicating the range of performance on these tasks.

\section{Discussion}

This difference between part-whole and part-part conditions supports the hypothesis that the relationship between the colored regions can play a role in visual search for color $\times$ color conjunctions. Wolfe et al. (1990) have reported results from 14 different color $\times$ color conjunction searches. All of these stimulus configurations produced target trial slopes of at least $20 \mathrm{msec} / \mathrm{item}$ and often much more. Blank trial slopes were at least twice the target trial slopes. In Experiment 1 of the present paper, however, part-whole color $\times$ color conjunction searches were performed with an efficiency comparable to other guided searches for conjunctions of color and orientation or color and size (see Treisman \& Sato, 1990; Wolfe et al., 1989, for examples). ${ }^{2}$ Obviously, something about the spatial distribution of colors allows for efficient search in one case but not in the other. A variety of hypotheses can be entertained: (1) There could be separate processors for the red color of the whole houses and the yellow color of the window parts. Combination of guidance from "part" and "whole" color processors would lead attention to the red house with yellow windows. (2) There could be a single parallel color processor and a separate processor of part-whole information. The task then becomes a more complex conjunction of (red $\times$ whole) $\times$ (yellow $\times$ part). (3) The task could be performed in two 
steps. First, information about red houses might become available in an analogue of the global precedence effect (Navon, 1977). Search through the set of red houses would follow as a second step. That search could be serial in the manner of Egeth et al. (1984), or it could be parallel in the manner of Friedman-Hill and Wolfe (1992), whose subjects searched for an odd orientation in a subset defined by color. In this scheme, there is a single color processor but the colors of wholes and the colors of parts must still not interfere with each other. In subsequent experiments, we attempted to eliminate some of these alternatives. For the present, it is clear that the part-whole stimuli used in Experiment 1 permit a deployment of attention that is far more efficient than any of the previous color $\times$ color conjunctions reported (Grabowecky \& Khurana, 1990; Wolfe et al., 1990).

\section{EXPERIMENT 2 Increasing Distractor Inhomogeneity}

When one sees the stimuli, one difference between the part-whole and part-part versions of Experiment 1 is quite clear. In the part-whole version, the houses seem to divide into a red group and a blue group. It then seems possible to perform a color feature search for yellow on the red group, ignoring the blue houses. In the part-part version, this "figure-ground" segmentation does not seem to work. Given this observation, there is a relatively uninteresting account for the results of Experiment 1. If we assume that the little yellow windows can be ignored in the initial grouping, the part-whole version has just two relevant colors (red and blue), whereas the part-part version has three (red, blue, and yellow). Part-part may be more difficult than part-whole simply because distractor heterogeneity makes search more difficult (see, e.g., Duncan \& Humphreys, 1989). One argument against this hypothesis is the finding that part-part color $\times$ color conjunction searches are very inefficient even when only two colors are present (Wolfe et al., 1990, Experiment 7). However, a true refutation requires a demonstration that part-whole color $\times$ color conjunctions can be done efficiently in the presence of three "house" colors. This was the purpose of Experiment 2.

\section{Method}

There were two conditions in Experiment 2. In both cases, the target was the red house with yellow windows. The stimuli were identical to those in the part-whole version of Experiment 1 with the addition of one distractor type. In Version 1, these were green houses with yellow windows. In Version 2, they were yellow houses with red windows. The three distractors were present in equal numbers. Both conditions increased distractor inhomogeneity. In Version 2, the color of the target part was used as the whole color for one of the distractors. This could further complicate the search. Does the presence of red in the windows make it harder to find red houses? Do yellow houses make it harder to find yellow windows? If Version 2 was not harder than the original part-whole condition of Experiment 1, it would become easier to argue that the color of wholes and the color of parts are somehow processed and/or represented separately. All other methods were the same as in Experiment 1. Ten new subjects were tested.

\section{Results and Discussion}

Figure 3 is a scatterplot of the individual target and blank trial slopes for Experiment 2. Part-whole and partpart data from Experiment 1 are shown for comparison. The Experiment 2 slopes are somewhat shallower than those for the part-whole version of Experiment 1 (Version 1, target trial average slope $=8.4 \mathrm{msec} / \mathrm{item}$,

Subject Slopes For Experiment Two

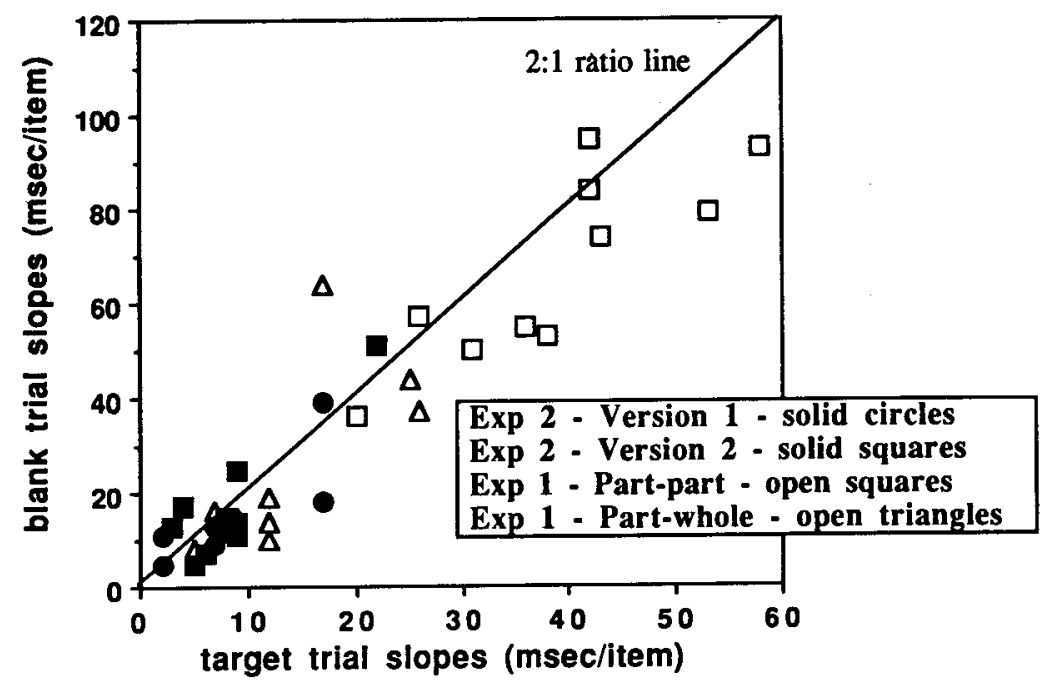

Figure 3. Scatterplot of individual slopes for four conditions of Experiment 3. Note that both conditions of Experiment 2 produce slopes that are shallower on the average than those of the part-whole condition of Experiment 1 and much shallower than those of the part-part condition of Experiment 1 . 


\section{Stimuli for Experiment Three}

Size irrelevant condition $\mathbf{A}$
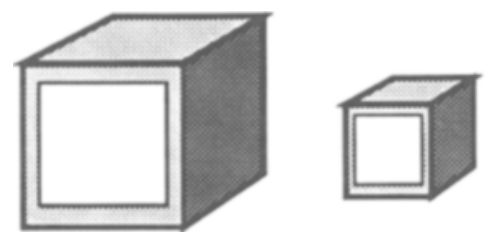

Red box with yellow face

\section{Size irrelevant condition B}
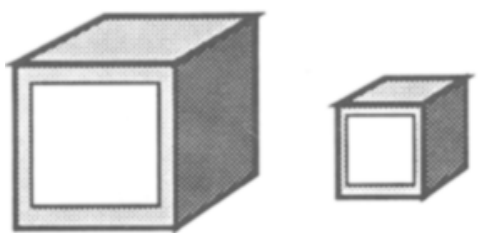

Red box with yellow face

\section{Big control condition}

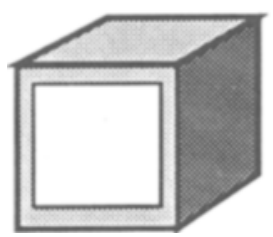

Red box with yellow face

\section{Little control condition}

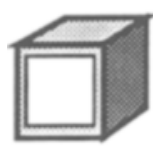

Red box with yellow face
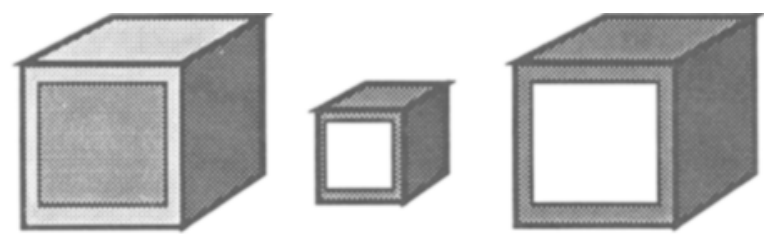

$\mathrm{red} / \mathrm{blue}$

blue/yellow

blue/yellow
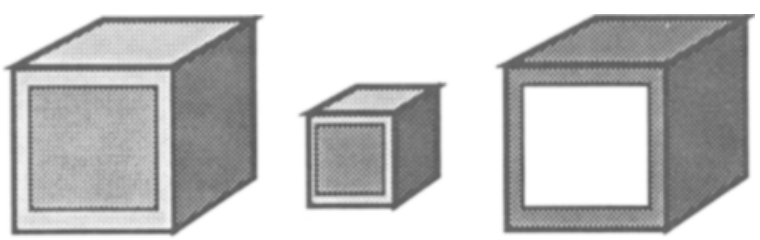

red/blue

$\mathrm{red} / \mathrm{blue}$

blue/yellow

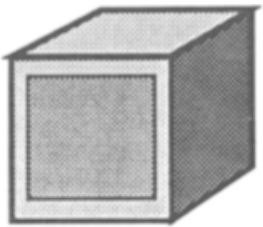

$\mathrm{red} / \mathrm{blue}$

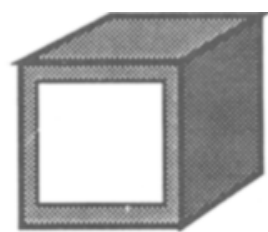

blue/yellow

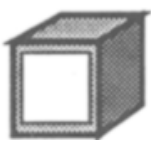

blue/yellow

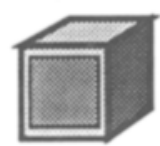

$\mathrm{red} / \mathrm{blue}$

Figure 4. Stimuli for Experiment 3.

blank trial $=14.7 \mathrm{msec} /$ item; Version 2 , target trial $=$ $8.3 \mathrm{msec} /$ item, blank trial $=17.0 \mathrm{msec} /$ item) . Both versions are much shallower than the part-part slopes of Experiment 1. These results, taken with the results from Wolfe et al. (1990), argue against distractor inhomogeneity as an account for the results of Experiment 1. Moreover, Version 2, with its yellow houses with red windows, shows that it is not critical that each target color be represented at only one scale. In Experiment 1, the only yellow regions were windows and the only red regions were houses. That constraint was eliminated in Version 2 of Experiment 2. Apparently, the presence of yellow houses did not disturb the search for yellow win- dows. If anything, search was more efficient than in the part-whole condition of Experiment 1. Most likely, this is due to the reduction of the number of red houses and/or the number of yellow windows from one half of the items to one third.

\section{EXPERIMENT 3}

The Effects of Physical Size

It might be suggested that efficient part-whole searches could be described as complex color $\times$ size searches. The part-whole conditions of Experiments 1 and 2 were all searches for a big red thing conjoined with a small yel- 
low thing. Size and part-whole information were perfectly correlated. To show that the part-whole information is implicated in the efficient searches of Experiments 1 and 2 , it must be shown that the task is not being done on the basis of size information. Experiments 3 and 4 show that the size relationships are not critical by showing that the "whole" need not be bigger than all of the "parts" in a display.

\section{Method}

In the previous experiments, the whole houses were always bigger than the windows. In Experiment 3, size and part-whole information were partially decoupled by using two sizes of targets and distractors. The stimuli were drawn so that the smaller "whole" target was smaller than the "part"' in the larger distractor. The stimuli are shown in Figure 4 . The task was a color $\times$ color conjunction search. The target was always the red cube (the whole) with a yellow square on the front surface (the part). (Some subjects labeled this "a red TV with a yellow screen.") There were two sizes of each stimulus. The small size was small enough to fit completely within the yellow square on the big size. The task can still be described as a search for a whole red thing with a yellow part. However, it was not possible to do the task as a complex conjunction of $($ big $\times$ red $) \times($ small $\times$ yellow), because on half the trials the big red target box was smaller than the small yellow square on the other half of the trials. Selection based on physical size would not be reliable.

Size-irrelevant condition A. Targets: big red cube with a yellow face, or small red cube with a yellow face. Distractors: big redblue, big blue-yellow, little blue-yellow.

Size-irrelevant condition $B$. Targets: big red cube with a yellow face, or small red cube with a yellow face. Distractors: big redblue, little red-blue, big blue-yellow.

Big control condition. Target: big red cube with a yellow face. Distractors: big red-blue, big blue-yellow.
Little control condition. Target: little red cube with a yellow face. Distractors: little red-blue, little blue-yellow.

Two distractor colors and two sizes create four types of distractor items. Since we were limited to three at a time, the size-irrelevant condition was split into two conditions each with three different distractors as described. Since the target was unpredictably big or small, the subjects could not search for conjunctions of color $\times$ size in these conditions.

Ten new subjects were tested. All other methods were similar to those in previous experiments.

\section{Results and Discussion}

Figure 5 gives a scatterplot of target and blank trial slopes for all subjects. It is clear that there are no significant differences between the conditions. Average target trial slopes were 16.0 and 13.3 for the two size-irrelevant conditions and 16.0 and 14.6 for the big and little control conditions, respectively. Average blank trial slopes were 33.3 and 28.6 for the two size-irrelevant conditions and 36.6 and 32.4 for the big and little control conditions. As can be seen in the scatterplot, these average slopes reflect a mix of subjects, some of whom produced apparently serial searches $(>20 \mathrm{msec} / \mathrm{item}$ on target trials, $>40$ on blank trials) and others of whom produced apparently guided searches (many with target slopes of $10 \mathrm{msec} /$ item or less).

Experiment 3 shows that variation in the size of items does not interfere with the efficiency of part-whole color $x$ color searches. This suggests that those searches are not complicated versions of color $\times$ size conjunction searches. Another approach to the same issue can be taken. In Experiment 4, part-part color $\times$ color stimuli

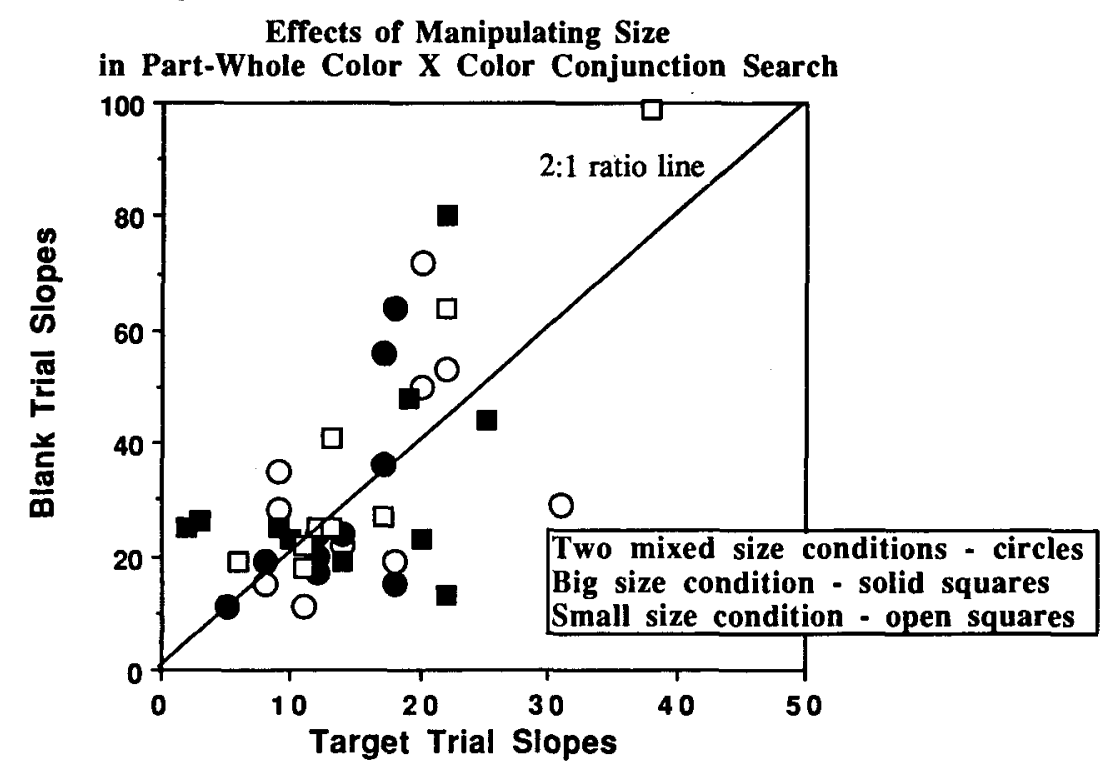

Figure 5. Scatterplot of individual slopes for four conditions of Experiment 2. Note that all conditions produce comparable results. 
were created with parts of very unequal size. If size information was the key to the efficiency of the part-whole conditions in the preceding experiments, one would expect the introduction of size information to improve search in a part-part condition.

\section{EXPERIMENT 4 \\ Part-Part Searches With Parts of Different Sizes}

\section{Method}

The stimuli were green cubes with one large and one small colored spot, as shown in Figure 6. The subjects searched for the cube with a large red and a small yellow spot among cubes with large red and small purple spots and cubes with large purple and small yellow spots. If size is useful, these could be described as searches for an item with big red and little yellow parts. In the one-face condition, both spots were on the front face of the cube. In the twoface condition, the small spot was moved to the side face. The 10 subjects from the preceding experiment were also tested here.

\section{Results and Discussion}

Average target trial slopes were $67.0 \mathrm{msec} /$ item in the one-face condition and 70 in the two-face condition. Blank trial slopes were 122 and 133, respectively. These are obviously highly inefficient searches and provide no support for the role of size in rendering color $\times$ color searches more efficient.

In Experiment 4, the two parts were spatially separated. This probably contributed to the difficulty of the task (Grabowecky \& Khurana, 1990), even though the dots were perceptually parts of a single object. The two-face condition was run in order to see whether there would be a difference when the dots were markings on different parts of the cube as opposed to when the marks were on a single part in the one-face condition. The manipulation made no difference; both tasks were very inefficient.

Perhaps it is unsurprising that the stimuli in Experiment 4 failed to support efficient, guided search. The task requires a conjunction of conjunctions, big red conjoined with small yellow, and there is no evidence elsewhere in the visual search literature for efficient search in this type of task. This makes it all the more interesting that it is possible to search for stimuli that can be described as a red whole conjoined with a yellow part. Although the partwhole distinction is intuitively appealing, "part"' and "whole" are labels of convenience. The results of the first

\section{Stimuli for Experiment Four}

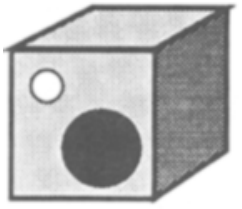

One Face

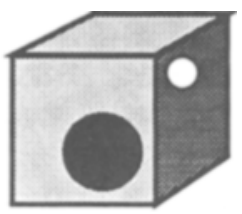

Two Face
Figure 6. Stimuli for Experiment 4. Can subjects search for the big red circle conjoined with the small yellow circle?
Target Stimuli for Experiment Five

The Effects of Surroundedness

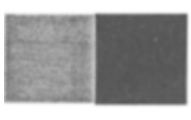

One Side

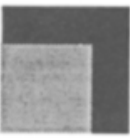

Two Sides

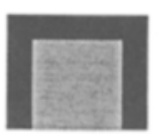

Three Sides

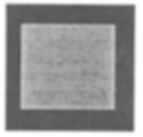

Four Sides
Figure 7. Stimuli for Experiment 5. All regions are the same area. All colors are equiluminous.

four experiments demonstrate that there is some parallel decomposition of items that can be used to support guided search for color $\times$ color conjunctions. A comprehensive account of that decomposition awaits further work. The remaining experiments presented in this paper will serve to illustrate some of the attributes of the decomposition that permit efficient color $\times$ color search.

\section{EXPERIMENT 5 The Role of Surroundedness}

What makes something a part? One contributing factor is the degree to which it is surrounded by the whole. The more one region is surrounded by another, the more it seems to be a part. Obviously, this is not a complete definition. An arm is a part of a body without being surrounded by the whole body.

\section{Method}

In an effort to examine the role of surroundedness, stimuli were created that varied in the degree to which one colored region surrounded the other. They are shown in Figure 7 . The target was always the blue and red item. The four conditions varied in the relationship of the red and blue regions. The red region could be bounded by blue on one, two, three, or four sides. The one-side condition was similar to the standard color $\times$ color tasks of Wolfe et al. (1990); the four-side condition was similar to the cubes in Experiment 3, though without any three-dimensional appearance. In all four conditions, the red and blue regions were of identical area (though, of necessity, not of identical linear extent). The distractors were red and yellow and blue and yellow items of the same shapes with the red and blue in the distractors occupying the same parts of items as in the target. In this experiment, to eliminate any role of a luminance cue, all three colors were made equiluminous by flicker photometry.

The experiment was otherwise identical to the previous experiments. Ten subjects were tested.

\section{Results and Discussion}

Figure 8 shows average slopes and slopes for individual subjects for target and blank trials, as a function of experimental condition. As the number of bounding sides increases, the slope decreases. This decrease was significant for both target and blank trials; in an analysis of variance (ANOVA), the main effects of condition and trial type and the interaction of condition and trial type were all significant at $p<.0001$, although contrasts between pairs of conditions suggested that the significant decrease was largely attributable to the decrease between the oneside and two-side conditions. Paired comparisons showed 

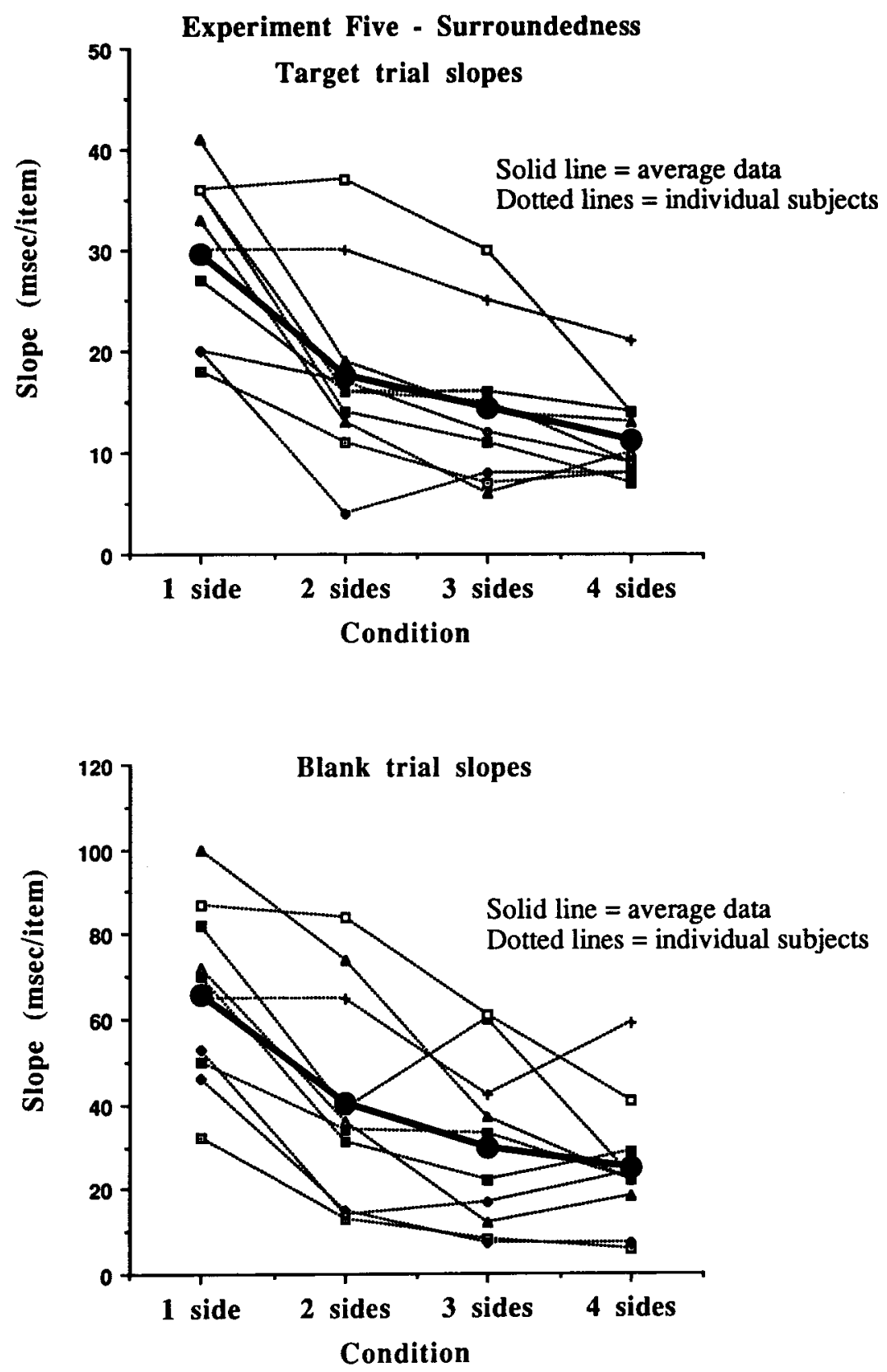

Figure 8. Results of Experiment 5 showing that as the part is more surrounded by the whole, search efficiency increases.

that for target trials, the one-side versus two-side conditions were significantly different $(F=12.3, p=.0012)$, whereas there was no significant difference for two versus three and three versus four sides. For the blank trials, one versus two sides and two versus three sides were significantly different $(F=54.4, p<.0001$, and $F=9.63$, $p=.004$, respectively); there was no significant difference for three versus four sides.

The results of Experiment 5 suggest that surroundedness contributes to the distinction between parts and wholes. Could surroundness be a complete account of these results? Some informal experimentation suggests not. Imagine a display divided into irregular regions of blue and green. On this background, red and yellow dots are displayed. The observer's task is to determine whether any red dot lies on a green portion of the background. In surroundedness terms, the target is a red dot surrounded by green. This is a very difficult search. Intuitively, the lack of discrete green items seems to be critical, but this is a topic that requires further experimentation. Although 
surroundedness by itself may not be sufficient to produce efficient color $\times$ color search, it may be necessary. We will return to this topic in the general discussion.

The use of equal areas for the two colors rules out an explanation of these results based on parts' being smaller in area than wholes unless subjects infer that the blue region continues underneath the red region in the two-, three-, and four-sided conditions. This would make the virtual blue (whole) object bigger than the red (part) object in the internal perceptual representation if not in the retinal image. The role of inference about occlusion and the unseen extent of parts and wholes is another interesting topic for future work (Enns \& Rensink, 1992).

The results of Experiment 5 also eliminate two other accounts of the comparative efficiency of part-whole color $x$ color conjunction searches. First, the tasks do not depend on some complicated conjunction of color and luminance information, since all colors had the same luminance in this experiment. Second, they make it clear that the figural and three-dimensional properties of the stimuli are not critical variables. That is, the whole does not have to look like a "house," a "cube," or any real, three-dimensional object. In this experiment, very simple two-dimensional stimuli produced effects similar to those seen with "houses" and "cubes."

Similar results were obtained in an experiment with stimuli like those of Experiment 4. Note that in Figure 5, the small dot can be moved so that it partially overlaps the large dot or so that it is entirely surrounded by the large dot. The more completely the small dot is surrounded by the large dot, the shallower the slopes of the RT $\times$ set size functions, suggesting again that surroundedness can define parts and wholes. This result also argues against the simple size hypothesis, since the size difference between the small and large regions decreases when the small region lies on top of the larger region.

\section{EXPERIMENT 6 The Definition of a "Part" Is Task Dependent}

The previous experiments show that some aspect of the spatial relationship between two colored regions permits efficient search for color $\times$ color conjunctions. Is this an entirely bottom-up, stimulus-driven analysis of the display, or can the same display be analyzed in two or more different ways? Intuition suggests that some aspects of the perceptual use of hierarchical relationships are under the control of the observer. Consider a room full of people. One could imagine searching for the man with the red flannel shirt or searching for the red flannel shirt with the Harvard Medical School insignia. In one case, the shirt is the "part." In the other, it is the "whole." Experiment 6 supports this intuition. With target stimuli held constant, an element that was designated a "part" in one condition could be treated as a "whole" in another con- dition, demonstrating that the definitions of "part" and "whole" are under substantial "top-down" control.

\section{Method}

The stimuli for the two conditions of Experiment 6 are shown in Figure 9. All stimuli were cubes with two concentric disks on their front surfaces. Both conditions of Experiment 6 were partwhole, color $\times$ color conjunction tasks. In the first disk $\times$ disk condition, the color of the cube was green and irrelevant. The target was the yellow disk with a red center. In the cube $\times$ disk condition, the color of the small central disk was irrelevant. The target was the red cube with the yellow disk on it. In the first condition, the yellow disk was the whole. In the second condition, it was a part. Efficient search in both conditions would indicate that the designation of part and whole could be modulated by task demands.

Ten subjects were tested. All other methods were similar to those of the previous experiments.

\section{Results and Discussion}

Average RT $\times$ set size functions are shown in Figure 10. It is clear that both of these tasks were quite efficient. Target trial slopes for individual subjects range from 1 to $15 \mathrm{msec} /$ item for the cube $\times$ disk condition and from 4 to $18 \mathrm{msec} /$ item for the disk $\times$ disk condition. The slopes do not differ significantly between the two conditions [target trials, $t(9)=1.6, p=.14$; blank trials, $t(9)=1.2, p=.27)$.

The results of this experiment make it clear that the definition of part and whole can be task dependent. The same yellow disk serves as the whole in the disk $X$ disk condition and as the part in the cube $x$ disk condition. Of course, this result points to the danger of using common words like part and whole to describe the internal workings of a visual process. The cube does not vanish in the disk $\times$ disk condition, and that cube remains, in some sense, a "whole." Nevertheless, it is clearly possible to use one of the several possible hierarchical relationships between the cube, the large disk, and the small disk, and it is possible for one element to serve the subordinate part role in one task and the superordinate whole role in another.

This ability to use part and whole labels operates under some constraints. For example, in Experiment 4, the subjects could not treat the big disk as the whole when it did not surround the smaller disk. In Experiment 5, the subjects could not take parts that abutted on only a single side and decide that one region was the whole and the other a part. We may conclude that part and whole assignments are neither arbitrary nor made in a strictly bottom-up, stimulus-driven fashion.

\section{GENERAL DISCUSSION}

Several conclusions may be drawn from this series of experiments. (1) Configural properties can make it possible to search efficiently for color $\times$ color conjunctions. (2) This ability is not a simple function of the relative sizes of colored regions. (3) All else being equal, efficient 


\section{Stimuli for Experiment Six: Color $\mathrm{X}$ color conjunctions}

\section{Disk X Disk Condition}

Conjunction of color of large disk (whole) with color of small disk (part). Color of cube is irrelevant. (Letters indicate color names)

Target

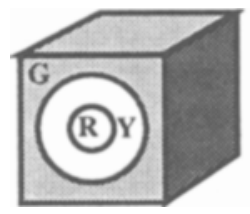

Distractors

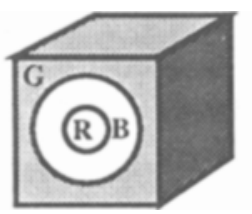

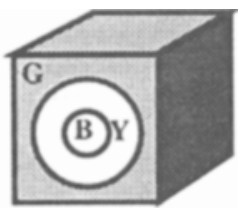

\section{Cube X Disk Condition}

Conjunction of color of cube (whole) with color of large disk (part). Color of small disk is irrelevant.

Target

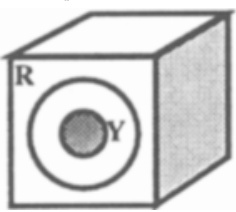

Distractors
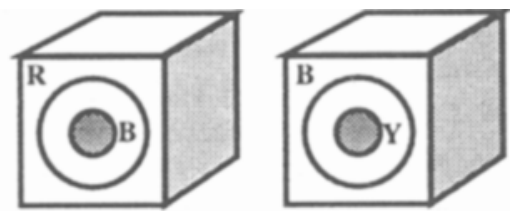

Figure 9. Stimuli for Experiment 6.

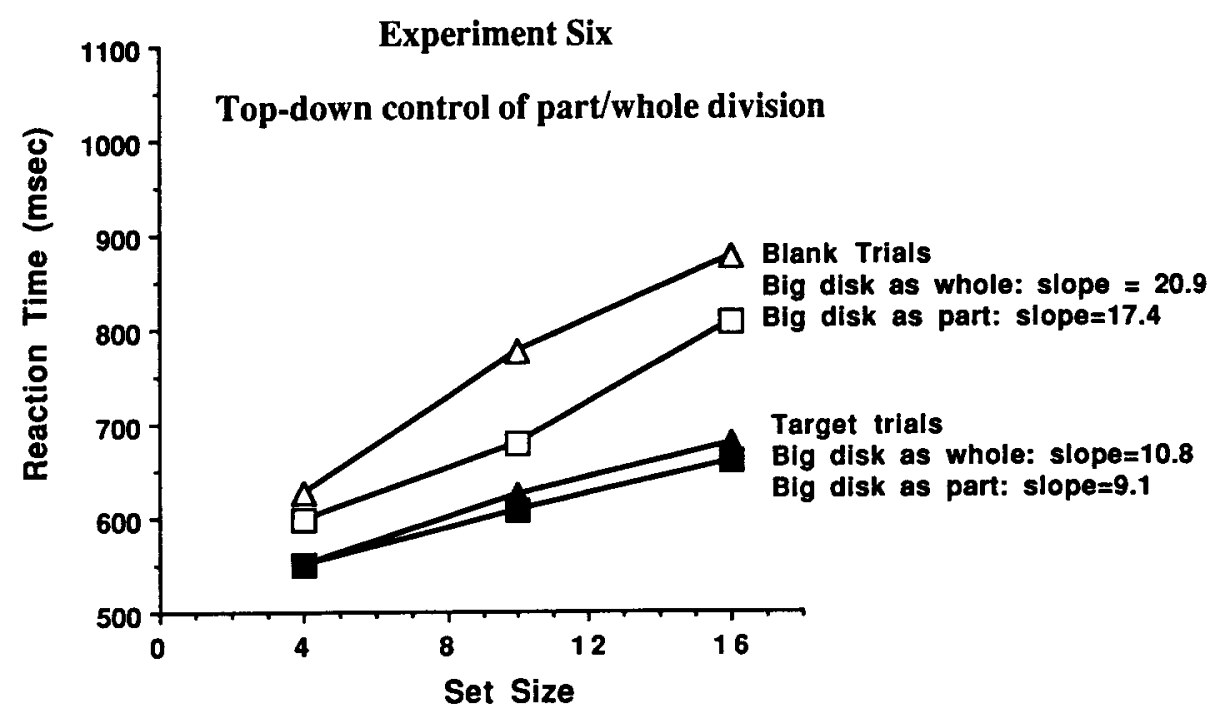

Figure 10. Results of Experiment 6, showing that subjects can select the same region to serve as the "part" in one task and the "whole" in another. 
search is more likely if one colored region is surrounded by another. (4) Subjects can choose to exploit one or another hierarchical relationship. This choice is constrained, but it is not under complete stimulus control.

Do the configural properties that allow for efficient color $\times$ color search have anything to do with the processing of parts and wholes, or is the part-whole terminology merely a convenient way to describe our stimuli? A definitive answer to this awaits further research, but we can speculate. Subjects often believe that the task was easy because the relevant set of "wholes" could be treated as a group. In Experiment 1, for example, it was easy to group all of the red houses. This grouping seems to have made it possible to determine whether one red house had a yellow window. The houses that were half red did not appear to group and search was hard. In Experiment 2, irrelevant houses with red windows did not group with relevant red houses and did not interfere with search. Another way to describe the grouping is to say that the red houses are being segregated into the figure, with all other houses relegated to the ground. Properties within the figure or group can be processed differently from properties elsewhere. ${ }^{3}$ Thus, it is possible to find the yellow windows in the group of red houses, somehow reducing (but not eliminating) interference from yellow windows elsewhere. If interference were completely eliminated, the search would be a trivial feature search for yellow and should proceed with near-zero slopes. If the results reported here are related to an ability to group items by color, this would argue against Treisman's conjecture that grouping does not occur for randomly placed items (Treisman, 1982).

The parallel stage of processing is usually envisioned as a set of relatively independent modules, each handling one basic feature and each apparently incapable of searching for more than one thing at a time. Even if one accepts the notion of parallel processing of some part-whole information, it is not completely clear how this part-whole distinction should be mapped onto this architecture and how it would permit search for two colors at once. The brute force approach would be to propose that separate modules exist for the color of wholes and the color of parts. There are several reasons to proceed cautiously in this direction. First, the wanton proliferation of modules should be avoided on grounds of parsimony. Second, as shown in Experiment 6, a "part" in one task may be a "whole" in another, indicating that any module would have to be very cognitively penetrable in ways that other modules (orientation, color, etc.) are not. Third, there is an alternative to separate part and whole modules that is not ruled out by the present data. If we take the color case as an example, perhaps red houses are identified by a color processing module. Their locations could be noted and the same color module could be used subsequently to look through the set of all marked locations for yellow windows. This two-step process could perform the task without the need for a second color module. Though a second color module would not be needed, the part-whole distinction is still needed. Otherwise it is hard to understand why this two-step process would not work for all color $\times$ color conjunctions. ("Mark the 'red' locations, then rerun the color module over the set of marked locations in a search for "yellow." ") In other tasks, in which two different features have been used, we have found evidence for two-step searches of this sort. Subjects can search through the red items in order to determine whether there is an item of odd orientation in that subset, ignoring nonred items (Friedman-Hill \& Wolfe, 1992). One argument against applying the two-step, subset search hypothesis to part-whole searches is that the pattern of RTs in subset searches is very different from the pattern in part-whole searches. When subjects search for the odd orientation in the red items, overall RTs are slower than RTs in standard guided searches for conjunctions. More significantly, blank trial RTs tend to be as fast as or faster than the target trial RTs, a pattern never seen in guided search for conjunctions and never seen in part-whole conjunction searches.

A plausible architecture might have separate but temporary parallel representations created for the colors of parts and wholes. A side effect of the representation of "wholes" might be the experience of the grouping of a set of items into a foreground figure. These representations would have to be flexible enough to change with task demands, and it is possible that they are only created in response to specific task demands. Even if part and whole representations are separate only in some transitory fashion, the central point of this paper remains: something like part-whole relations can be exploited "preattentively." A concrete version of this hypothesis might be based on the work of Robertson and Lamb (1991). They suggest that global properties are preferentially processed in the right hemisphere, and local, in the left. Perhaps color part-part searches cause two colors to compete for processing resources in the left hemisphere, whereas part-whole searches allow for a more efficient division of labor.

The idea that visual attention can be deployed over whole objects (rather than over locations, for example) is certainly not new. For example, Treisman and Kahneman introduced the idea of object files that would gather the information about the various attributes of an object and preserve the object over various transformations (Kahneman \& Treisman, 1984; Treisman, 1988; see also Kanwisher \& Driver, 1992). Tipper and his colleagues have conducted a series of elegant experiments, pitting location against object and finding that attention can be deployed to one object even if two are at the same location (Tipper, 1992; Tipper et al., 1990; Tipper et al., 1991). In a particularly clear illustration of the role of objects, Baylis and Driver (1993) found that two parts of an image could be more readily compared to each other if they formed part of a single object than if they were each part of a different object. Our experiments add a hierarchical structure to the descriptions of objects in visual attention. In Treisman and Kahneman's terms, one might 
say that object files list not only the color, size, orientation, etc. of an object. They can also maintain the distinction between the color of the object as a whole and the color of a constituent part.

The experiments in this paper are restricted to the color domain. Can part-whole relationships be exploited in other feature domains? We have investigated orientation and size. For size, it is indeed the case that the search for the big whole with the little part is easier than the search for the item with big and little parts. The pattern of results is similar to that shown here for color. A series of size $\times$ size, part-whole experiments will be reported in a subsequent paper. For orientation, the situation is quite different. We have found no case in which the search for a part-whole stimulus is significantly more efficient than a search for a comparable part-part stimulus. Thus, a search for a vertical whole with an oblique part is no easier than a search for an item with vertical and oblique parts. Searches for form $\times$ form conjunctions (e.g., search for the $S$ made of Xs among Ss made of Os and Os made of Xs) follow orientation and are, likewise, inefficient.

One way to think about the distinction between color and size on the one hand and orientation and form on the other is to note that color and size are relatively independent of frame effects (if we ignore, for now, the effects of color and size contrast and constancy). Unlike orientation and form, color and size do not change when the head is rotated or when the object is rotated. Thus, a big red book with a horizontal title remains big and red under rotation, but the horizontal title will change orientation and the letter will become harder to read. The experiments on orientation will be the substance of another paper.

In summary, the experiments presented here show that visual search tasks can be influenced by part-whole relationships. Parallel processes can be used to guide attention to an item defined by the conjunction of the color of the whole item and the color of a part of that item, just as attention can be guided to an item defined by the conjunction of the color and orientation of an item. By contrast, attention cannot be guided to an item defined by the conjunction of the color of one part of an item with the color of another part. These results add to the picture of the parallel stage of visual processing. Although there are severe limitations on parallel processing in the visual system, there is clear evidence for some quite sophisticated processing beyond the mere extraction of basic visual features.

\section{REFERENCES}

Baylis, G. C., \& Driver, J. (1993). Visual attention and objects: Evidence for hierarchical coding of location. Journal of Experimental Psychology: Human Perception \& Performance, 19, 451-470.

BEN-AV, M. B., SAGI, D., \& BraUN, J. (1992). Visual attention and perceptual grouping. Perception \& Psychophysics, 52, 277-294.

Bravo, M., \& Blake, R. (1990). Preattentive vision and perceptual groups. Perception, 19, 515-522.

CAVE, K. R., \& WolfE, J. M. (1990). Modeling the role of parallel processing in visual search. Cognitive Psychology, 22, 225-271.
Chun, M. M., \& Wolfe, J. M. (1994). Just say no: How are visual searches terminated when there is no target present? Manuscript submitted for publication.

DehaENE, S. (1989). Discriminability and dimensionality effects in visual search for featural conjunctions: A functional pop-out. Perception \& Psychophysics, 46, 72-80.

DUNCAN, J. (1989). Boundary conditions on parallel processing in human vision. Perception, 18, 457-469.

DunCAN, J., \& Humphreys, G. W. (1989). Visual search and stimulus similarity. Psychological Review, 96, 433-458.

D'Zmura, M. (1991). Color in visual search. Vision Research, 31, 951-966.

Egeth, H. E., Virzi, R. A., \& Garbart, H. (1984). Searching for conjunctively defined targets. Journal of Experimental Psychology: Human Perception \& Performance, 10, 32-39.

Enns, J. T., OCHS, E. P., \& Rensink, R. A. (1990). VSearch: Macintosh software for experiments in visual search. Behavior Research Methods, Instruments, \& Computers, 22, 118-122.

ENNS, J. T., \& ReNsink, R. A. (1992). An object completion process in early vision. Investigative Ophthalmology \& Visual Science, 33, 1263. (Abstract)

Friedman-Hill, S., \& Wolfe, J. (1992). Activation vs inhibition in visual search. Investigative Ophthalmology \& Visual Science, 33, 1356.

GraboweCKY, M., \& KhuRANa, B. (1990). Features were meant to be integrated. Investigative Ophthalmology \& Visual Science, 31, 105.

Humphreys, G. W., \& Muller, H. (1993). Search via recursive rejection (SERR): A connectionist model of visual search. Cognitive Psychology, 25, 43-110.

Kahneman, D., \& Treisman, A. (1984). Changing views of attention and automaticity. In R. Parasuraman \& J. Beatty (Eds.), Varieties of attention (pp. 29-61). Orlando, FL: Academic Press.

KANwiSher, N., \& Driver, J. (1992). Objects, attributes, and visual attention: Which, what, and where. Current Directions in Psychological Science, 1, 26-31.

KinCHLA, R. A., \& Wolfe, J. M. (1979). The order of visual processing: "Top-down," "bottom-up," or "middle-out." Perception \& Psychophysics, 25, 225-231.

Kwak, H. W., Dagenbach, D., \& Egeth, H. (1991). Further evidence for a time-independent shift of the focus of attention. Perception \& Psychophysics, 49, 473-480.

LAMB, M. R., \& RoberTSON, L. C. (1990). The effect of visual angle on global and local reaction times depends on the set of visual angles presented. Perception \& Psychophysics, 47, 489-496.

Lennie, P., Trevarthen, C., VanEssen, D., \& Wassle, H. (1990). Parallel processing of visual information. In L. Spillman \& J. S. Werner (Eds.), Visual perception: The neurophysiological foundations (pp. 103-128). San Diego: Academic Press.

MCLeod, P., Driver, J., \& CRISP, J. (1988). Visual search for conjunctions of movement and form is parallel. Nature, 332, 154-155.

NAGY, A. L., \& SANCHEZ, R. R. (1990). Critical color differences determined with a visual search task. Journal of the Optical Society of America A, 7, 1209-1217.

Nakayama, K., \& Silverman, G. H. (1986). Serial and parallel processing of visual feature conjunctions. Nature, 320, 264-265.

NAvON, D. (1977). Forest before the trees: The precedence of global features in visual perception. Cognitive Psychology, 9, 353-383.

Pavel, M., Econopouly, J., \& LANdy, M. S. (1992). The psychophysics of rapid visual search. Investigative Ophthalmology \& Visual Science, 33, 1355.

Quinlan, P. T., \& HumphreYs, G. W. (1987). Visual search for targets defined by combinations of color, shape, and size: An examination of the task constraints on feature and conjunction searches. Perception \& Psychophysics, 41, 455-472.

Robertson, L. C., EgLY, R., Lamb, M. R., \& Kerth, L. (1993). Spatial attention and cuing to global and local levels of hierarchical structure. Journal of Experimental Psychology: Human Perception \& Performance, 19, 471-487.

Robertson, L. C., \& LAMB, M. R. (1991). Neuropsychological contributions to theories of part/whole organization. Cognitive Psychology, 23, 299-330. 
Ross, W. D., Grossberg, S., \& Mingolla, E. (1993). A neural model of visual search. Investigative Ophthalmology \& Visual Science, 34, 1235.

TIPPER, S. P. (1992). Selection for action: The role of inhibitory mechanisms. Current Directions in Psychological Research, 1, 105-108.

TIPPER, S. P., BreHAut, J. C., \& DrIVER, J. (1990). Selection of moving and static objects for the control of spatially directed attention. Journal of Experimental Psychology: Human Perception \& Performance, 16, 492-504.

Tipper, S. P., Driver, J., \& Weaver, B. (1991). Object centered inhibition of return of visual attention. Quanerly Joumal of Experimental Psychology, 43A, 289-298.

TrEisMan, A. (1982). Perceptual grouping and attention in visual search for features and for objects. Journal of Experimental Psychology: Human Perception \& Performance, 8, 194-214.

TREISMAN, A. (1986a, November). Features and objects in visual processing. Scientific American, pp. 114-125.

Treisman, A. (1986b). Properties, parts, and objects. In K. R. Boff, L. Kaufmann, \& J. P. Thomas (Eds.), Handbook of human perception and performance (pp. 35.1-35.70). New York: Wiley.

Treisman, A. (1988). Features and objects: The 14th Bartlett Memorial Lecture. Quarterly Journal of Experimental Psychology, 40A, 201-237.

Treisman, A., \& Sato, S. (1990). Conjunction search revisited. Journal of Experimental Psychology: Human Perception \& Performance, 16, 459-478.

Venturino, M., \& GaGnon, D. A. (1992). Information tradeoffs in complex stimulus structure: Local and global levels in naturalistic scenes. Perception \& Psychophysics, 52, 425-436.

WoLFE, J. M. (1992). The parallel guidance of visual attention. Current Directions in Psychological Science, 1, 125-128.

WolfE, J. M. (1994). Guided Search 2.0: A revised model of visual search. Manuscript submitted for publication.

Wolfe, J. M., CAVe, K. R., \& Franzel, S. L. (1989), Guided search: An alternative to the feature integration model for visual search. Journal of Experimental Psychology: Human Perception \& Performance, $15,419-433$.

Wolfe, J. M., Yee, A., \& Friedman-Hill, S. R. (1992). Curvature is a basic feature for visual search. Perception, 21, 465-480.
Wolfe, J. M., Yu, K. P., Stewart, M. I., Shorter, A. D., Friedman-Hill, S. R., \& Cave, K. R. (1990). Limitations on the parallel guidance of visual search: Color $x$ color and orientation $X$ orientation conjunctions. Journal of Experimental Psychology: Human Perception \& Performance, 16, 879-892.

Zohary, E., \& HochsteIN, S. (1989). How serial is serial processing in vision? Perception, 18, 191-200.

\section{NOTES}

1. The part-whole terminology used here seems obviously related to the previously described ability to process information at different scales (global and local properties). Previous work in this area has generally focused on the effects of one scale on another, or on the possibility that one scale has precedence over another (Kinchla \& Wolfe, 1979; Lamb \& Robertson, 1990; Navon, 1977; Robertson, Egly, Lamb, \& Kerth, 1993; Venturino \& Gagnon, 1992).

2. In these "guided" searches, as in many others, blank to target trial slope ratios are near 2.0. The 2:1 slope ratio is a hallmark of a serial self-terminating search. However, a serial self-terminating search through the entire set of items is not the only search that will produce a $2: 1 \mathrm{ra}-$ tio. Consider a search for a red T among red and green Ls. Search for a T among Ls is generally serial, but this search will be a serial search through just the red letters. There would be no point in searching through the greens. The resulting $\mathrm{RT} \times$ set size functions will have the $2: 1$ slope ratio characteristic of serial self-terminating search, but the search is better described as "guided" because half of the distractors are eliminated by parallel guidance from the color module. This is generally true in guided search. The parallel processes reduce the effective set size by guiding attention toward likely targets. Search is serial but through a subset of the items.

3. In several recent studies (Ben-Av, Sagi, \& Braun, 1992; Bravo \& Blake, 1990) and models of visual search (Humphreys \& Muller, 1993; Ross, Grossberg, \& Mingolla, 1993) extensive use has been made of grouping as a way to facilitate search.

(Manuscript received March 16, 1993; revision accepted for publication October 9, 1993.) 